\title{
Percepción estudiantil de la importancia de la retroalimentación
}

\section{Students 'perception of the importance of feedback:} from valorization to the rubrics use

\section{CÉSAR RAFAEL NARVÁEZ CARRIÓN*, (ID MIGUEL ÁNGEL VERDEZOTO CARRERA (D)}

\author{
Universidad Estatal de Bolívar \\ Av. Ernesto Che Guevara s/n y Av. Gabriel Secaira, Guaranda - Ecuador
}

*cnarvaez@ueb.edu.ec

\section{RESUMEN}

El aprendizaje de un idioma extranjero representa un desafío en más de un aspecto, y la evaluación en el proceso no es la excepción. La gran cantidad de estudiantes, con su multiplicidad de intereses y aptitudes, son un aspecto a tomarse en cuenta en el diseño de actividades a fin de lograr su inclusión en el proceso y con ello mantener su motivación. En este sentido, la retroalimentación por parte de los docentes es un factor fundamental en el mencionado proceso, como lo demuestran numerosos estudios investigativos que abordan las múltiples facetas de esta compleja temática. Esta realidad plantea la incógnita de si los estudiantes, actores principales del proceso de aprendizaje, son conscientes de la importancia de la retroalimentación para su óptimo aprendizaje.

Palabras clave: Retroalimentación, técnica de evaluación, retroinformación, proceso de aprendizaje.

\section{ABSTRACT}

The learning of a foreign language is a challenge in more than one sense and its assessment in the process is not the exception. 
The large number of students with their variety of interests and aptitudes is an aspect to consider when designing activities in order to get real inclusion in the process and consequently to keep their motivation high. In this sense, teachers' feedback is a key factor in the aforementioned process, as it is shown by many research studies, which tackle multiple aspects of this complex subject. This reality sets the question about whether or not the main actors of the process, the students, are aware of the importance of feedback for improving their learning in a better way.

Keywords: Feedback, evaluation techniques, learning process.

\section{INTRODUCCIÓN}

La percepción de la retroalimentación por parte del estudiante y su frecuencia de uso son aspectos poco investigados en el proceso de enseñanza aprendizaje. Existe escasa literatura acerca del punto de vista del estudiante, incluso en la educación superior, a raíz de un enfoque centrado en el docente. De verificarse dicha importancia, se ratifica lo necesario de un enfoque más andragógico y conocer las expectativas de los estudiantes para un mayor impacto y una práctica más eficaz.

El presente estudio busca corroborar la importancia de la retroalimentación para un proceso de aprendizaje más eficaz y pretende motivar la organización del tiempo a fin de obtener una retroalimentación continua y personalizada.

En este sentido, existen múltiples consideraciones en cuanto a la utilidad de la retroalimentación para el desarrollo de las destrezas de aprendizaje. Esta compleja realidad de interés pedagógico andragógico motiva el presente estudio al plantear las siguientes incógnitas: ¿̇Los estudiantes reconocen la importancia de la retroalimentación dentro de su proceso de aprendizaje del idioma inglés? y ¿̇Existe una aplicación generalizada de la estrategia de retroalimentación en la Universidad Estatal de Bolívar?

A partir de estas preguntas se asume como hipótesis: Si la investigación y el estudiantado coinciden en la importancia de los procesos de retroalimentación, dicha estrategia será ampliamente aplicada para un aprendizaje óptimo de un idioma extranjero.

Alineándose a la verificación de dicha hipótesis, ajustarse a un principio de objetividad significa prevenir el actuar a voluntad en la instrucción en el aula (Hua-yan, 2001) y centrarse en el análisis de la retroalimentación para llegar a un ejercicio de escritura en torno a dicha retroalimentación y un análisis más profundo de la misma luego de constatar que, cuando el estudiante no se siente involucrado, la productividad del proceso de retroalimentación decae significativamente (Muncie, 2000). De allí la importancia de la conciencia del estudiante acerca del valor de la retroalimentación, una de las interrogantes del presente artículo.

Dentro de la enseñanza, evaluar el grado de retención y aprendizaje en los estudiantes, ha sido el pilar fundamental que permite evaluar la metodología aplicada. Estos son aspectos importantes ya que el docente tiene que manejar grupos diversos en aptitudes y costumbres, y al mismo tiempo en diversos tipos de inteligencias. Así, poder diseñar nuevos métodos que permitan converger dichos talentos y facilitar el aprendizaje 
de los estudiantes (Jung-Ho Jung y Don-Ryun Chang, 2018), tanto en el proceso como en la evaluación, resulta un asunto de gran relevancia en la actualidad, subrayando la importancia de una actitud más activa de los estudiantes. Este último punto se muestra como un pilar fundamental para incrementar la motivación intrínseca en los estudiantes, un factor altamente influenciado por el rol docente y el uso de metodologías efectivas no solamente en general, sino específicamente en el contexto ecuatoriano (Auquilla et al, 2019).

Entre esos métodos es necesario incluir el uso de nuevas tecnologías disponibles en la enseñanza, mismas que permiten hacer el proceso de aprendizaje más dinámico involucrando al estudiante en la clase. Pérez-Segura et al. (2020) reportan la utilidad del uso de tecnología nueva en clase como factor de motivación. Al mismo tiempo, su investigación demuestra cómo el trabajo basado en una retroalimentación personalizada contribuye significativamente al desarrollo de sus destrezas receptivas. En el contexto ecuatoriano se han podido evidenciar ciertas prácticas como el uso de wikis para incrementar la motivación y lograr mejores desempeños (Del Pezo González, 2015) y la incorporación de las redes sociales para obtener beneficios como la integración del idioma en su vida cotidiana (Comina Tayo, 2016).

Por otro lado, Bruton (2007) se centra en la comunicación escrita y reporta el uso de retroalimentación guiada por medio de pistas para apuntalar el proceso de enseñanza, provocando reflexión y guiando el trabajo del estudiante en lugar de dar una corrección directa y concluye con los resultados de mejora por parte de la muestra de estudiantes.
Por su parte, Ghosn-Chelala y Al-Chibani (2018) enriquecen el panorama significativamente al incorporar la retroalimentación grabada en video en lugar del tradicional comentario escrito, con lo cual se respalda la necesidad de reservar tiempo para valorar el trabajo de cada estudiante, lo cual una vez más fundamenta la ventaja de tener clases con menor cantidad de estudiantes para la obtención de mejores resultados.

Ahora bien, independientemente del formato, Ni (2012) asegura que la retroalimentación tiene un impacto positivo sobre los factores afectivos y promueve las prácticas de retroalimentación para lograr mejores resultados en el proceso de aprendizaje basándose en la teoría de Filtro afectivo de Stephen Krashen, considerando los aspectos de autoestima, motivación y ansiedad. Este aspecto puede ser complementado con los hallazgos de Toro et al. (2019), quienes reportan un aumento en la ansiedad del estudiantado cuando se enfrentan a un entorno reducido de aprendizaje del idioma en donde el control y el seguimiento por parte del docente serían mayores.

Además de las distinciones entre retroalimentación oral y escrita, Li (2013) investiga el impacto de dos modalidades de retroalimentación: implícita y explícita, concentrándose en dos componentes particulares, la habilidad analítica del idioma y la memoria de trabajo. Si bien su estudio se enfoca en el aprendizaje del idioma chino, su enfoque es aplicable a la dinámica del aprendizaje de idiomas extranjeros en general.

De esta manera, las consideraciones sobre el valor de la retroalimentación para promover un aprendizaje de mayor 
calidad y la problemática detectada al ignorar la percepción de los estudiantes, por un lado, y la frecuencia de aplicación de dicha práctica por parte del cuerpo docente, dan forma a los objetivos de la presente investigación: Conocer la percepción de los estudiantes sobre la retroalimentación y a la vez constatar con qué frecuencia lo aplican los docentes de la institución.

\section{MATERIALES Y MÉTODOS}

Una vez tomados en cuenta los diferentes referentes teóricos de los que ya se ha hablado en la primera parte, se llevó a cabo el presente estudio siguiendo la siguiente metodología:

Se procedió al muestreo probabilístico por conveniencia para investigar el impacto de la retroalimentación en el desempeño académico y en la motivación estudiantil. Los datos analizados se obtuvieron a través de la aplicación de una encuesta digital a una muestra de 361 estudiantes pertenecientes a los tres niveles (A1, A2 y B1) del Departamento de Idiomas de la Universidad Estatal de Bolívar. A continuación, se analizan los resultados obtenidos en dicha encuesta.

\section{RESULTADOS}

En el siguiente gráfico se analiza el grado de conocimiento de la población estudiantil sobre la retroalimentación, con una escala de 1 a 5 . Donde 5 representa tener una idea clara y 1 no tiene idea.

\section{Figura 1}

Grado de conocimiento de retroalimentación

150

100

50

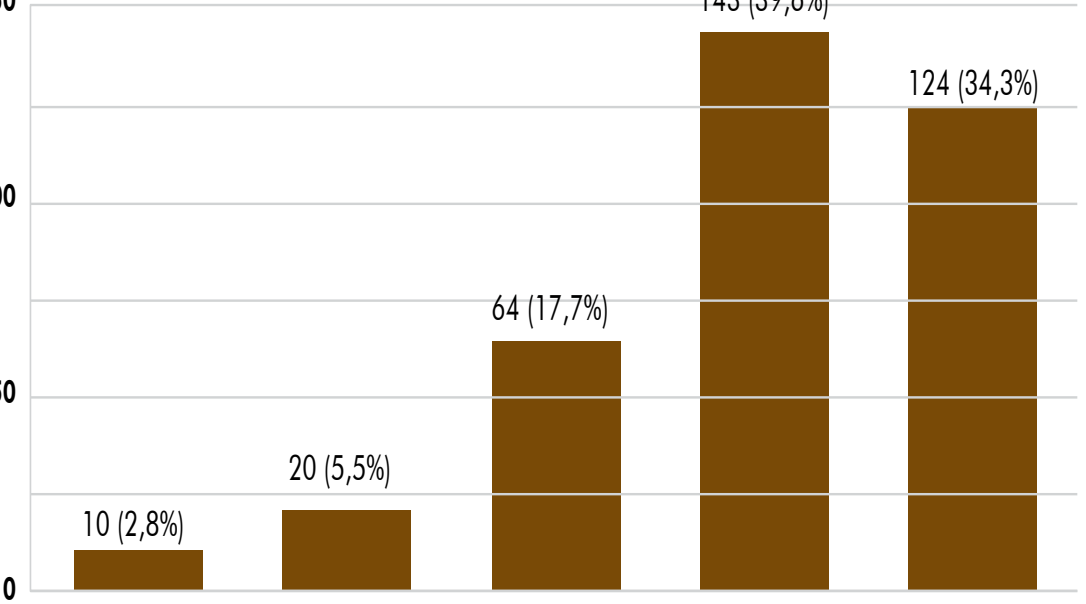

1

3

4

5

Nota. Gráfico donde se analiza el grado de conocimiento de la población estudiantil sobre la retroalimentación, con una escala de 1 a 5.

Al observar los resultados se puede notar que el $73.9 \%$ tiene conocimiento de qué es una retroalimentación, un $17.7 \%$ se encuentra en duda, y $2.8 \%$ absolutamente no tiene idea o no ha trabajado con una retroalimentación. Este conocimiento por parte de los estudiantes evidencia la implementación de dicha práctica por parte de los docentes de la Universidad Estatal de Bolívar.

Analizando en mayor detalle los resultados obtenidos, 2/3 de la población conoce la técnica, esto es favorable al considerar que por lo menos en una ocasión han sido expuestos y por ende 
están familiarizados con su uso, facilitando así su aplicación en futuras ocasiones. Entonces, esto nos permitirá comprobar si la manera de abordar la materia es la adecuada o existe un campo para mejorar.

A su vez, los resultados obtenidos en este punto demuestran que los docentes de la Universidad Estatal de Bolívar son conscientes de la utilidad de la retroalimentación para mejorar la calidad de la enseñanza y la aplican de alguna manera en los procesos de enseñanza-aprendizaje. Si bien, como se verá más adelante, la frecuencia de aplicación es un punto a mejorar, un aspecto que se alinea con lo investigado

\section{Figura 2}

Grado de importancia de la retroalimentación para el estudiante

300

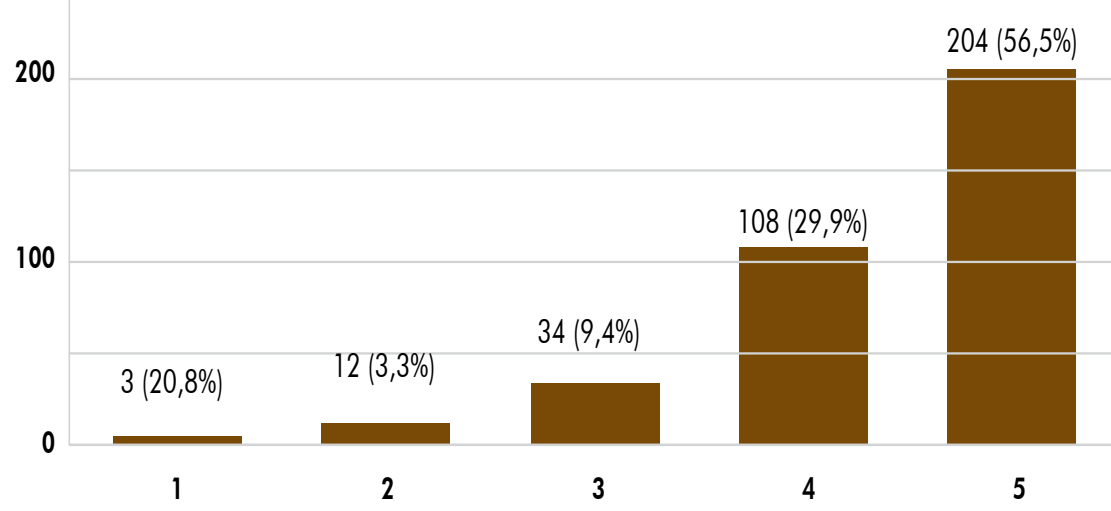

Nota. Gráfico donde se analiza la variable de la importancia de la retroalimentación para el estudiante. por Jung-Ho Jung y Don-Ryun Chang (2018) puesto que, si la evaluación requiere un lugar central en el proceso de aprendizaje, los procesos de retroalimentación no pueden dejar de ser parte de la misma.

En la Figura 2 se analiza la variable de importancia de la retroalimentación para el estudiante, en donde 1 significa que no tiene importancia y 5 que es muy importante.

En comparación con el cuadro 1, se observa una predisposición hacia recibir retroalimentación, lo cual sustenta la hipótesis planteada sobre la importancia del uso de la retroalimentación en el proceso de enseñanza en donde el $86.4 \%$ de los estudiantes están dispuestos a recibir una retroalimentación. Es decir, hay el espacio para aplicar nuevos métodos de enseñanza que permitan hacer el entorno educativo más dinámico.

Considerando las preguntas que originaron el presente estudio, las evidencias de este trabajo muestran la importancia que los estudiantes conceden a la retroalimentación y la utilidad de la misma para mejorar la dinámica de aprendizaje en términos generales, así como en el ámbito de los idiomas extranjeros.

En contraparte, el enfoque desde la docencia hacia su uso - aplicación se encamina no solamente para afianzar la metodología en el proceso de aprendizaje, sino también para mejorar la autoestima por parte del estudiante. Este es un aspecto de vital importancia, como lo demuestran los estudios de investigadores como $\mathrm{Ni}$ (2012) en cuanto a la utilidad de la retroalimentación para mejorar la dinámica de aprendizaje. 


\section{Figura 3}

Frecuencia de uso de retroalimentación

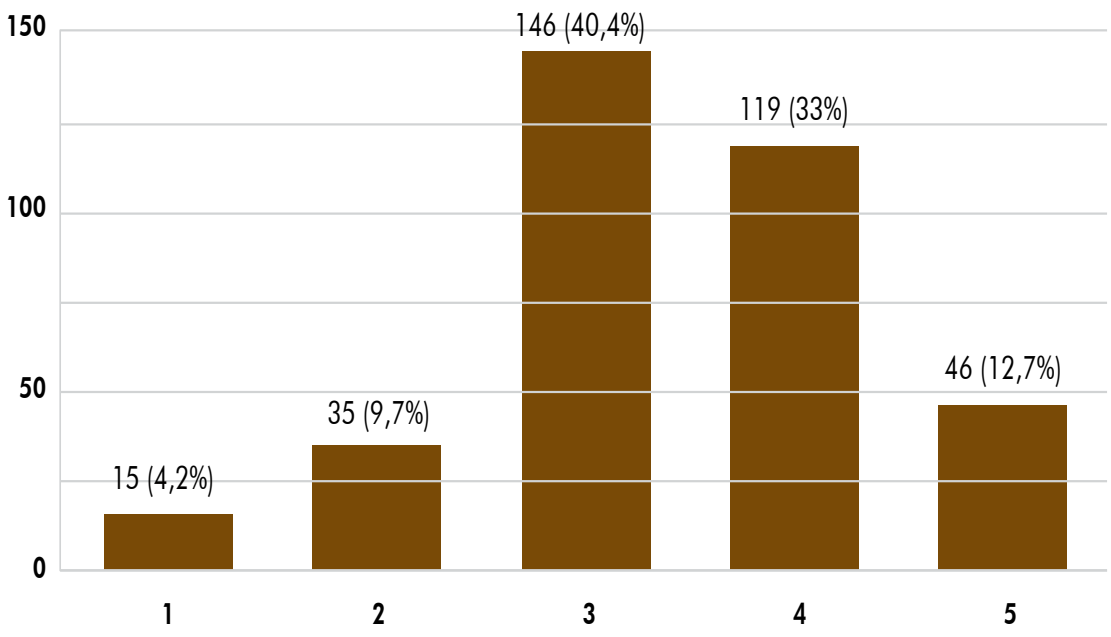

Nota. Gráfico donde se analiza la variable de frecuencia de recepción de retroalimentación por parte del docente.

En la Figura 3 se analiza la variable de frecuencia de recepción de retroalimentación por parte del docente, en donde 1 representa que el estudiante no la ha recibido, y 5 que siempre ha recibido una retroalimentación. Así, en respuesta a la pregunta número dos, se constata la utilización de la retroalimentación en el proceso de aprendizaje, un aspecto a potenciarse a través de las estrategias adecuadas para ello.

Al observar los resultados obtenidos en la Figura 3, el 40.4 $\%$ de la población en estudio, manifiesta haber recibido una retroalimentación en ocasiones, mientras que el $45.7 \%$ manifiesta que lo recibe frecuentemente. Al comparar estos una brecha importante que no está siendo cubierta por parte del docente y que tiene un impacto directo en la calidad de educación impartida. Estos resultados reflejan un aspecto a mejorar de gran importancia si se toma en cuenta las conclusiones de Jung-Ho Jung y Don-Ryun Chang (2018) acerca de la importancia de la evaluación y de la retroalimentación para un óptimo aprendizaje.

\section{Figura 4}

Importancia de la retroalimentación en el mejoramiento del lenguaje

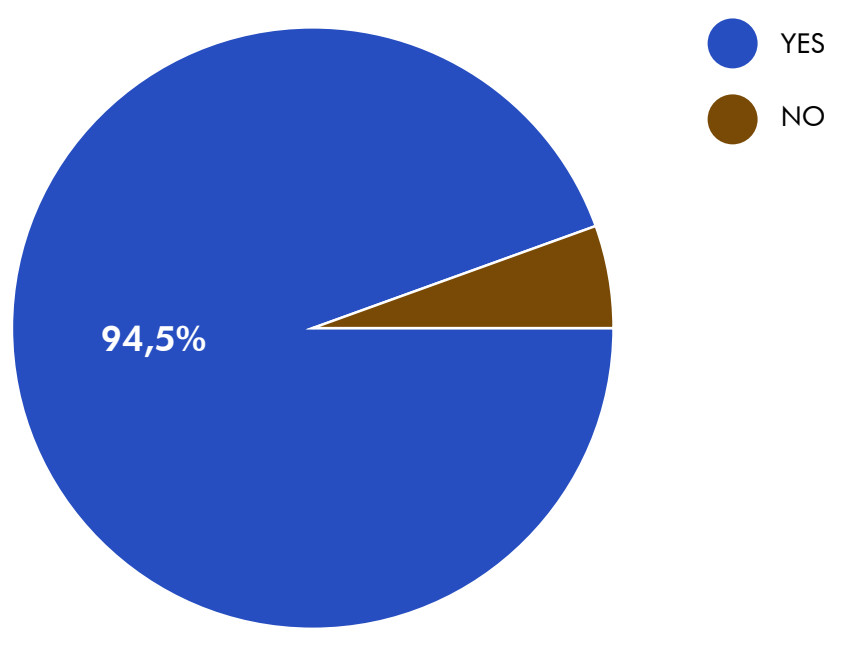

Nota. El $94.5 \%$ de los estudiantes encuestados considera que es importante el uso de la retroalimentación para mejorar o facilitar el aprendizaje de un nuevo idioma.

El $94.5 \%$ de los estudiantes encuestados considera que es importante el uso de la retroalimentación para mejorar o facilitar el aprendizaje de un nuevo idioma, respaldando los resultados obtenidos previamente en cuanto a la importancia 
de la retroalimentación por parte el estudiantado. Esto corrobora los estudios de Hyland (2010) acerca de la utilidad de la retroalimentación para mejorar los mecanismos de autorregulación y evaluación de su propio desempeño.

Adicionalmente, es importante considerar que el grado de autoestima y confianza en sí mismo del estudiante facilita su aprendizaje de un nuevo idioma. El recibir una retroalimentación objetiva que le permita monitorear su progreso va a impactar de manera favorable en el proceso de enseñanza y en la calidad del ambiente de clase, aspectos clave si tomamos en cuenta la investigación previa de $\mathrm{Ni}$ (2012) sobre la incidencia de la retroalimentación en el aspecto emocional.

\section{Figura 5}

Preferencia de recepción de retroalimentación

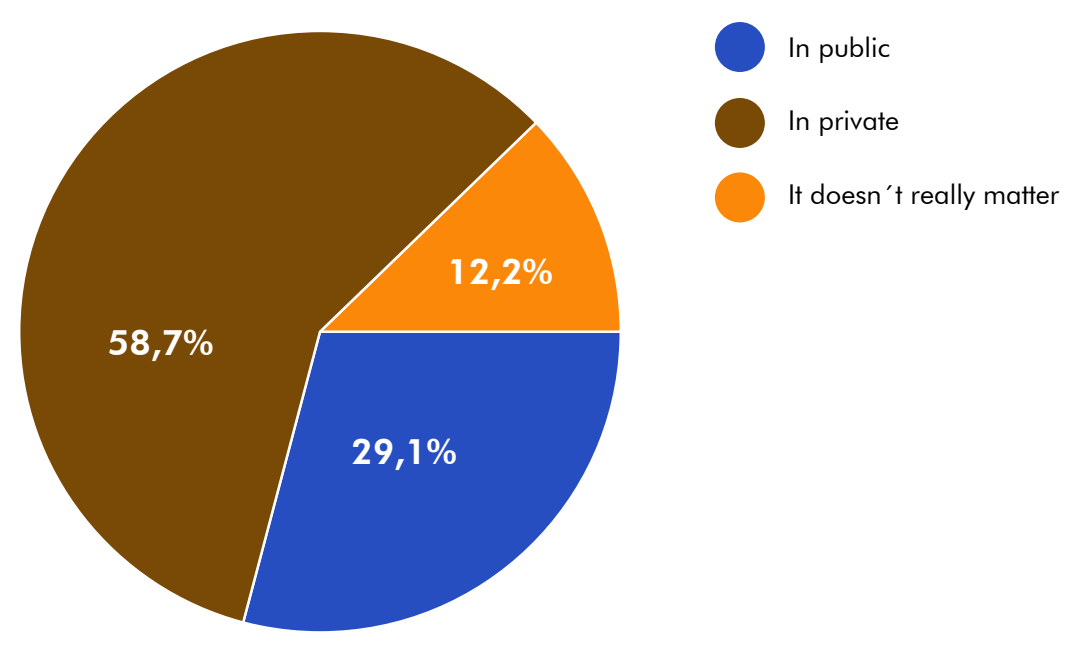

Nota. El $58.7 \%$ de los estudiantes prefieren recibir una retroalimentación en privado, esta cifra ratifica la importancia del diseño de rúbricas para el proceso de evaluación.
El $58.7 \%$ de los estudiantes prefieren recibir una retroalimentación en privado, esta cifra ratifica la importancia del diseño de rúbricas para el proceso de evaluación ya que, dada la cantidad de estudiantes por grupo, en la mayoría de casos no es posible dar una retroalimentación de uno en uno. Para ello es primordial el uso de dichas herramientas que permitan hacer una corrección del error de manera puntual y evitar las asociaciones directas de los errores con los estudiantes.

De ahí se desprende la necesidad de desarrollar la empatía por parte del docente. Es importante concientizar a la comunidad universitaria sobre la necesidad de implementar un manejo de clase eficaz sin caer en el autoritarismo y respetando en todo momento al estudiante, como caracteriza al quehacer educativo por su dimensión formativa. En palabras de Feshbach y Feshbach (2009), la empatía del docente desembocará en un mayor entendimiento y aceptación por parte del estudiante, desarrollando así actitudes más positivas hacia el aprendizaje. Un aspecto a investigarse con mayor profundidad.

Considerando que el 83.1 \% de los estudiantes prefieren recibir una retroalimentación directa, al aplicar una observación sobre el error cometido se estaría llegando a más de 4/5 del total de la población, esto implica un impacto significativo en favor de mejorar el proceso de enseñanza, en donde le sea posible al docente mitigar el hecho de tener que trabajar con grupos numerosos de estudiantes, sin tener que sacrificar la calidad de la enseñanza. 


\section{Figura 6}

Preferencia de la retroalimentación

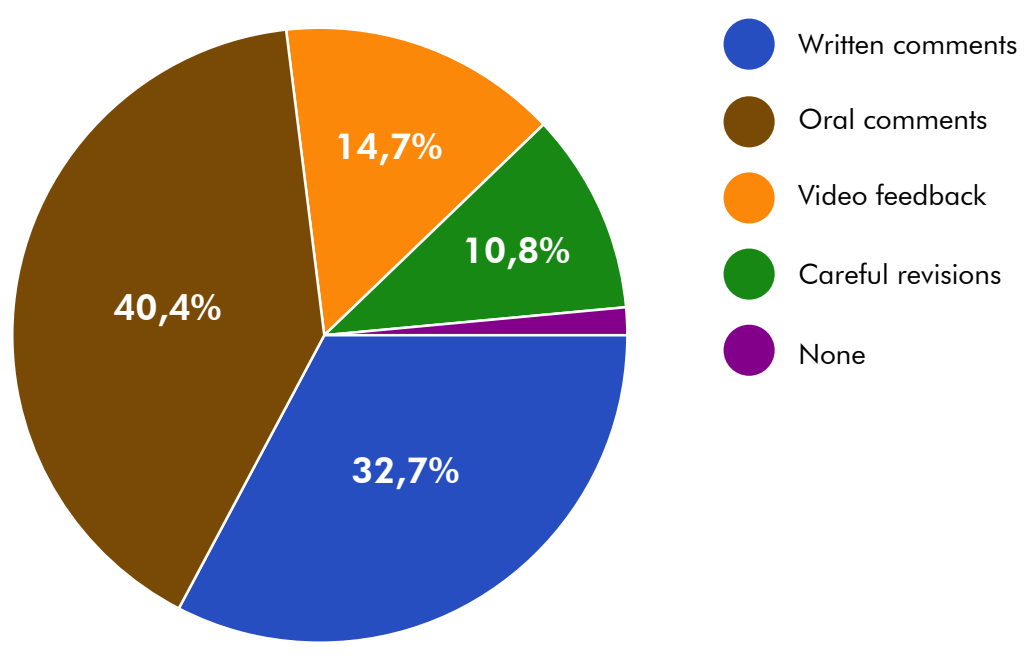

Nota. Técnicas adecuadas para lograr la retroalimentación de todos los estudiantes de manera personalizada.

Es importante implementar técnicas adecuadas para lograr la retroalimentación de todos los estudiantes de manera personalizada como sugiere la presente investigación y una amplia literatura previa: "Proveer retroalimentación inmediata e individual a los estudiantes es un factor crítico para mejorar la adquisición de destrezas y de conocimiento en la educación superior" (Serral et al., 2016, p.1). En este aspecto es donde la enseñanza tiene un campo a desarrollar, es importante orientar todas las interacciones para que contribuyan a un mejor desempeño a sabiendas de que un manejo adecuado del tiempo es un manejo eficaz de las interacciones entre colegas y con instructores, un trabajo colaborativo real, y un alto grado de compromiso (Alaslani y Alandejani, 2020).

\section{DISCUSIÓN}

En respuesta a la interrogante principal de la presente investigación, los resultados obtenidos muestran la plena conciencia de los estudiantes acerca del valor de la retroalimentación constante para mejorar su proceso de desarrollo de las distintas destrezas en el proceso de aprendizaje de idiomas extranjero. Dichos hallazgos confirman la base de la andragogía centrada en el estudiante, que lo hace consciente y activo dentro de su proceso de aprendizaje una vez minimizadas sus inhibiciones y orientados sus comportamientos, problemáticas mencionadas por Conner (1997) al compararla con pedagogía.

La información recabada sustenta lo constatado por Vargas (2007), en cuanto a la relación estudiante-profesor, una relación que, a decir de los estudiantes, debería caracterizarse por lo afectivo y lo académico (Castejón y Pérez, 1998), uno de los determinantes institucionales asociados al rendimiento académico estudiantil.

Adicionalmente, considerando la interrogante segunda, se evidencia la existencia de dicha práctica cuya frecuencia media en el proceso de aprendizaje y puede explicarse por las variables de carácter institucional, particularmente la cantidad de alumnos por clase Nargas, 2007), lo cual a su vez fundamenta la práctica objetiva mediante la aplicación de instrumentos de evaluación como las rúbricas.

\section{CONCLUSIONES}

Como conclusiones, dando respuesta a las preguntas generadoras de la presente investigación podemos observar que: 
1. Los estudiantes manifiestan conocer lo que es la retroalimentación y reconocen su valor como mecanismo de apoyo en el proceso de aprendizaje, en general y particularmente en el aprendizaje del idioma inglés, lo cual demuestra la aplicación de esta herramienta por parte de los docentes de la Universidad Estatal de Bolívar.

2. La frecuencia de aplicación es un aspecto de gran importancia y, como se pudo constatar en el presente estudio, puede incrementarse para lograr mejores resultados. En este sentido, se espera evidenciar la disposición favorable, mencionada con anterioridad, para motivar la investigación y la implementación de estrategias y herramientas que permitan un aumento en la práctica de la retroalimentación con miras a una mejora en el proceso de aprendizaje.

Además, el grado de conciencia de los estudiantes acerca del valor de la retroalimentación para lograr mejores resultados en el proceso de aprendizaje evidencia la utilidad de la herramienta en mención para estimular el grado de compromiso de docentes y estudiantes en una dinámica más centrada en el estudiante. Ya que los mismos se manifiestan como habituados a recibir retroalimentación pues se reporta dicha estrategia como una práctica generalizada en las aulas de la universidad.

Finalmente, los resultados en cuanto a la preferencia de la retroalimentación oral reflejan la importancia del diálogo en el aula universitaria, en consonancia directa con las dinámicas de andragogía con la valoración del estudiante y de su rol activo en el proceso de enseñanza aprendizaje.

\section{AGRADECIMIENTOS}

El presente estudio de investigación se aplicó gracias a la colaboración de los diferentes actores del proceso de enseñanza-aprendizaje en el seno de la Universidad Estatal de Bolívar. En tal virtud cabe una mención particular a la población de estudiantes cuya participación facilitó la información que se analiza en el presente estudio.

\section{REFERENCIAS}

Alaslani, K., \& Alandejani, M. (2020). Identifying factors that influence students performance through social networking sites: An exploratory case study. Heliyon, 6(4), e03686.

Auquilla, D. P. O., Maggi, M. R. T., Camacho, C. S. H., \& Fernández, R. A. (2019). Factors that influence Ecuadorian university students' motivation towards English learning: An exploratory research study. Dominio de las Ciencias, 5(2), 736-766.

Bruton, A. (2007). Vocabulary learning from dictionary referencing and language feedback in EFL translational writing. Language Teaching Research, 11 (4), 413-431.

Castejón, C., \& Pérez, S. (1998). Un modelo causal-explicativo sobre la influencia de las variables psicosociales en el rendimiento académico. Revista Bordon. Sociedad Española de Pedagogía. 2(50), 170-184.

Comina Tayo, I. M. (2016). The social network Facebook as a supporting tool for the English language skills development with students of tenth years of basic education at Pujilí technical high school during the academic cycle April-August 2015 (Bachelor's thesis, LATACUNGA/UTC/2016). 
Conner, M. L. (1997). Andragogy and pedagogy. Ageless learner, 2004, $01-18$.

Del Pezo González, G. (2015). Wikis as a teaching tool to improve english academic reading skill on adult of eighth grade at colegio particular mixto bachillerato a distancia Prof. Ernesto González Muñóz, La Libertad, province of Santa Elena, school year 20152016 (Bachelor's thesis, La Libertad; Universidad Estatal Península de Santa Elena, 2015.).

Feshbach, N. D., \& Feshbach, S. (2009). Empathy and education. The social neuroscience of empathy, 85, 98.

Ghosn-Chelala, M., \& Al-Chibani, W. (2018). Screencasting: supportive feedback for EFL remedial writing students. The International Journal of Information and Learning Technology.

Hua-yan, L. U. (2001). Principle of Objectiveness in the Classroom Instruction of Second Language. Journal of Beijing Normal University (Social Science Edition), (6), 16.

Hyland, F. (2010). Future directions in feedback on second language writing: Overview and research agenda. International Journal of English Studies, 10(2), 171-182.

Jung-Ho J., Don-Ryun Ch., (2018). Types of creativity-Fostering multiple intelligences in design convergences talents. Thinking Skills and creativity. Volume 23, March 2017, Pgs $101-111$.
Li, Shaofeng (2013). "The interactions between the effects of implicit and explicit feedback and individual differences in language analytic ability and working memory." The Modern Language Journal 97. 3: 634-654.

Muncie, J. (2000). Using written teacher feedback in EFL composition classes.

$\mathrm{Ni}$, Hui. (2012) "The effects of affective factors in SLA and pedagogical implications." Theory \& Practice in Language Studies 2.7

Pérez-Segura, J. J., Sánchez Ruiz, R., González-Calero, J. A., \& CózarGutiérrez, R. (2020). The effect of personalized feedback on listening and reading skills in the learning of EFL. Computer Assisted Language Learning, 1-23.

Serral, E., De Weerdt, J., Sedrakyan, G., \& Snoeck, M. (2016, June). Automating immediate and personalized feedback taking conceptual modelling education to a next level. In 2016 IEEE Tenth International Conference on Research Challenges in Information Science (RCIS) (pp. 1-6). IEEE.

Toro, L. V., Pinza, E. I., Vargas, A. B., Zuniga, A., Paredes, F. M., \& Espinosa, F. O. (2019). Teachers and Students' Perceptions of Teaching and Learning English in Small Classes: A Case of Ecuador. Turkish Online Journal of Educational Technology-TOJET, 18(1), 95-104.

Vargas, G. M. G. (2007). Factores asociados al rendimiento académico en estudiantes universitarios, una reflexión desde la calidad de la educación superior pública. Revista Educación, 31 (1), 43-63. 


\section{BIOGRAFÍA DE LOS AUTORES}

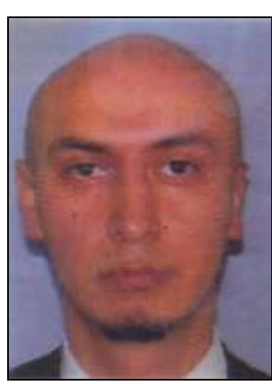

CÉSAR RAFAEL NARVÁEZ CARRIÓN, MSC.

Magíster en Pedagogía de Enseñanza del Idioma Inglés y Licenciado en Ciencias de la Educación, Mención Inglés por la Universidad Técnica Particular de Loja. Experiencia como profesor de idiomas inglés y francés en escuelas, colegios y universidades, tanto en el sector público como en el privado

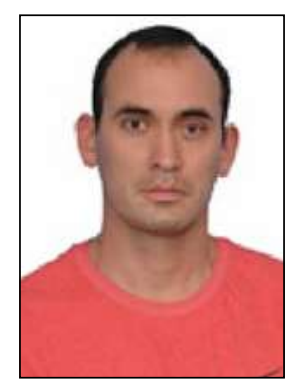

MIGUEL ÁNGEL VERDEZOTO CARRERA, ING.

Docente Universitario con amplia experiencia en educación. Graduado Zamorano, viaja posteriormente a Estados Unidos y trabaja en distintas áreas: granjas lecheras, industria productos cosméticos y del hogar, y en el sector financiero. De regreso en Ecuador labora en proyectos del Buen Vivir y como docente universitario de idioma extranjero. 